\title{
Ferromagnetic Micromechanical Magnetometers
}

\author{
Jack W. Judy, Henry Yang, Nosang Myung, Pedro Irazoqui-Pastor, \\ Morton Schwartz, Ken Nobe and Ken Yang \\ Electrical Engineering Department 1 , Biomedical Engineering Department 2 , \\ Chemical Engineering Department ${ }_{3}$ of the University of California, Los Angeles \\ Los Angeles, CA 90095-1594
}

\begin{abstract}
A novel micromechanical magnetometer is introduced that integrates electroplated cobalt-based magnetic materials with surface micromachined polysilicon structures. This new magnetometer has favorable scaling properties (both size and power) when compared to existing magnetic sensing technologies. Theoretical device performance is compared with experimental results with good agreement. These first prototype devices achieve a scale-independent performance of $100 \mu \mathrm{T} / \mathrm{mrad}$.

\section{INTRODUCTION}

We have designed, fabricated, and tested novel low-power ferromagnetic micromechanical magnetometers. The need for miniature high-sensitivity low-power magnetometers is driven by applications such as wireless sensor networks, which are useful for monitoring and controlling large areas, large facilities, and large numbers of mobile assets [1]. Table 1 compares the sensitivity, power consumption, and the scaling properties of the sensitivity with both dimension and power consumption of several magnetometer technologies (adapted from [2]).

Search-coil magnetometers have the advantage of being low power (all power is consumed by sense electronics), but their sensitivity scales down with area, making miniaturization counter productive. Flux-gate magnetometers have a high sensitivity, but it also scales down rapidly as it is miniaturized. Although semiconductor-based magnetometers, such as Hall plates, magnetotransistors, and magnetodiodes, are very compact, the sensitivity of these magnetometers is not competitive.
\end{abstract}

Table 1. Comparison of magnetometer sensitivity, power consumption, and scaling properties (Adapted from [1]).

\begin{tabular}{|c|c|c|c|c|}
\hline Magnetometer & $\begin{array}{c}\text { Sensitivity } \\
\text { Range } \\
\text { (Tesla) }\end{array}$ & $\begin{array}{c}\text { Dimensional } \\
\text { Scaling } \\
\text { (Sensitivity) }\end{array}$ & $\begin{array}{c}\text { Power } \\
\text { Range } \\
\text { (W) }\end{array}$ & $\begin{array}{c}\text { Power } \\
\text { Scaling } \\
\text { (Sensitivity) }\end{array}$ \\
\hline Search Coil & $\begin{array}{c}10 \mathrm{pT} \\
(\mathrm{MHz})\end{array}$ & $\mathrm{s}^{-2}$ & $\begin{array}{c}\text { Limited by } \\
\text { sense } \\
\text { circuitry }\end{array}$ & $\mathrm{p}^{0}$ \\
\hline Hall Effect & $10 \mu \mathrm{T}$ & $\mathrm{s}^{0}$ & $10^{-3}-10^{-4}$ & $\mathrm{p}^{-1 / 2}$ \\
\hline Semiconductor & $1 \mu \mathrm{T}$ & $\mathrm{s}^{0}$ & $10^{-3}-10^{-4}$ & $\mathrm{p}^{-1 / 2}$ \\
\hline MR & $10 \mathrm{pT}$ & $\mathrm{s}^{0}$ & $10^{-5}-10^{-7}$ & $\mathrm{p}^{-1 / 2}$ \\
\hline SQUID & $10 \mathrm{fT}$ & $\mathrm{s}^{0}$ & $\begin{array}{c}10^{1} \\
\text { (cryo) }\end{array}$ & $\mathrm{p}^{0}$ \\
\hline $\begin{array}{c}\text { MEMS } \\
\text { (Resonant) }\end{array}$ & $100 \mathrm{pT}$ & $\mathrm{s}^{-1}$ to -2 & $10^{-2}-10^{-5}$ & $\mathrm{p}^{-1 / 2}$ \\
\hline $\begin{array}{c}\text { Ferromagnetic } \\
\text { MEMS-Based } \\
\text { Magnetometer }\end{array}$ & $100 \mathrm{pT}$ & $\mathrm{s}^{0}$ & $\begin{array}{c}\text { Limited by } \\
\text { sense } \\
\text { circuitry }\end{array}$ & $\mathrm{p}^{0}$ \\
\hline
\end{tabular}

*Theoretical performance with circuits designed for inertial microsensors. Magnetoresistance magnetometers have a high sensitivity, but it scales as $p^{-1 / 2}$ with power due to a linear dependence on drive voltage. Although SQUID magnetometers are the most sensitive magnetometers, they require an extensive support system (cryorefrigeration unit). Recently Lorentz-force based resonant micromechanical magnetometers have been developed that perform well, but suffer from the fact that their sensitivity is scaled down with both dimension and power dissipation (i.e., driving current) and their output is a strong function of $\mathrm{Q}$, thus requiring hermetic packaging [3-4].

The magnetometer that we have designed, fabricated, and tested is a novel MEMS-based magnetometer that is capable of a high sensitivity that does not scale down with dimension and consumes no power other than that needed for the sense circuits.

\section{THEORY}

The concept behind the operation of this low-power magnetometer is straightforward: a micromechanical compass. Figure 1 illustrates the basic operation of the MEMS compass. An ambient magnetic field $(H)$ will produce a torque

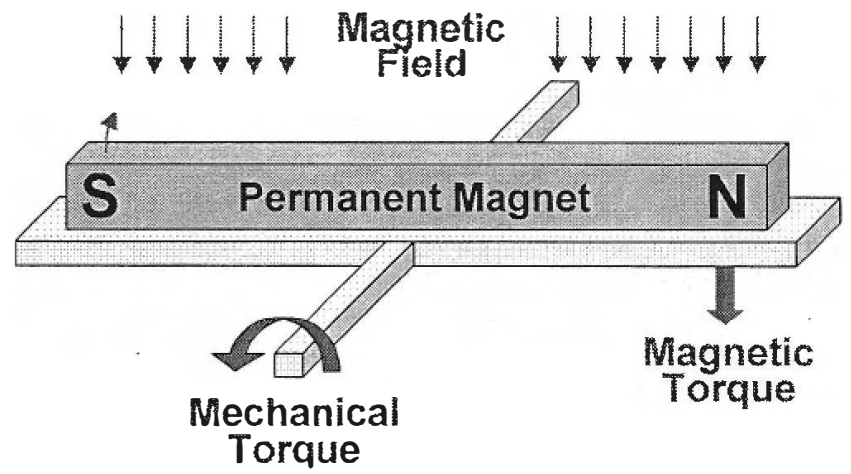

Figure 1. Schematic representation of the principle of operation of the ferromagnetic MEMS magnetometer.

$$
T_{\text {mag }}=(M \times H) \cdot v
$$

on a permanent magnet with a magnetization $M$. The magnetic torque is then transferred to a torsional microflexure, which creates an equal and opposite mechanical torque

$$
T_{m e c h}=k_{\phi} \cdot \phi
$$

with angular deflection $\phi$ and angular stiffness $k_{\phi}$. For a torsion bar with a rectangular cross section, the angular stiffness is proportional to

$$
k_{\phi} \sim a^{3} \cdot b \cdot G / l
$$

with shear modulus $G$, torsion bar length $l$, and cross section dimensions $a$ and $b$ where $b \geq a$. By equating Eq. 1 and Eq. 2 the angular deflection can be solved for, which yields

$$
\phi=(M \times H) \cdot v / k_{\phi} .
$$

Despite the fact that the magnetic torque (Eq. 1) scales down with a cubic dependence, so does the angular stiffness of the torsion bar (Eq. 3). The result is that the ratio of the two, and hence the angular deflection produced by the sensed magnetic field, has no dependence on dimensional scaling (Eq. 4). 


\section{EXPERIMENTAL DETAILS}

Our first prototype devices consist of a polysilicon micromechanical structure, produced with the MUMPs foundry service [5], and a Co-based ferromagnetic film that is electroplated onto the polysilicon plate (Figure 2 illustrates one example). In previous work by our group and others on magnetic microactuators, the electroplated ferromagnetic materials integrated with silicon-based flexures were magnetically soft (i.e., characteristically with a low coercive magnetic field $H_{c}$ ). However, often the best ferromagnetic material for microactuator and microsensor applications is a permanent magnetic material (i.e., characteristically with a high coercive magnetic field). To integrate the cobalt-based magnetic material onto the MUMPs chips, we used the following steps: resist removal, oxide etch to expose polysilicon, adhesion layer $(\mathrm{Cr})$ and seed-layer $(\mathrm{Ni})$ deposition, electroplating mask photolithography, electrically conductive packaging for electroplating, resist removal, seed-layer removal, final release oxide etch, and lastly supercritical drying.

The devices tested consisted of dual $4.2 \times 2 \times 200 \mu^{3}$ polysilicon torsion bars and a $100 \times 100 \times 13.4 \mu \mathrm{m}^{3}$ ferromagnetic plate. The magnetic properties of the electroplated Co-Ni material have been characterized in both a full-film form, as is typically done, and in the actual geometry tested (i.e., the B-H loop of the $100 \times 100 \times 13.4 \mu \mathrm{m}^{3} \mathrm{Co}-\mathrm{Ni}$ plate was measured individually with a vibrating-sample magnetometer to extract the coercivity and remnant magnetization for the theoretical model). The response of these prototype devices to an ac magnetic field was measured with a laser doppler vibrometer. A plot of the angular deflection as a function of magnetic field is given in Figure 3. Although these results clearly demonstrate functionality and feasibility, we anticipate that future devices will integrate capacitive displacement sensing circuits, borrowed from the inertial microsensor industry. The ability to use integrated sense circuitry will provide the opportunity to detect sub- $\AA$ displacements, which would translate into detecting a $\mu \mathrm{T}$-level of magnetic flux with the current device geometry. By improving the remnant magnetization and changing the geometry of the magnet to $100 \times 100 \times 13.4 \mu \mathrm{m}^{3}$ the magnetometer with integrated sense circuitry could detect a nT-level of magnetic flux.

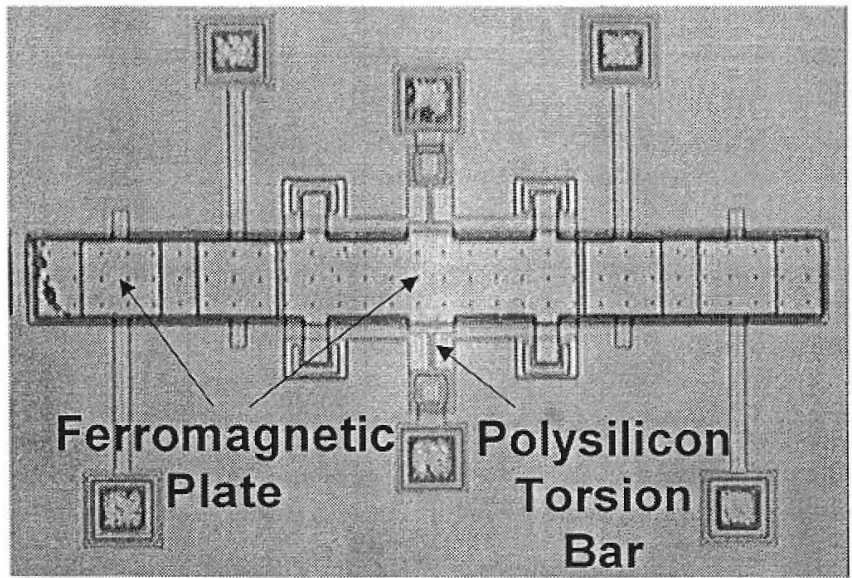

Figure 2. Optical image of a magnetometer with Co-Ni magnetic material electroplated on top of a polysilicon micromechanical structure made with MUMPS [5].

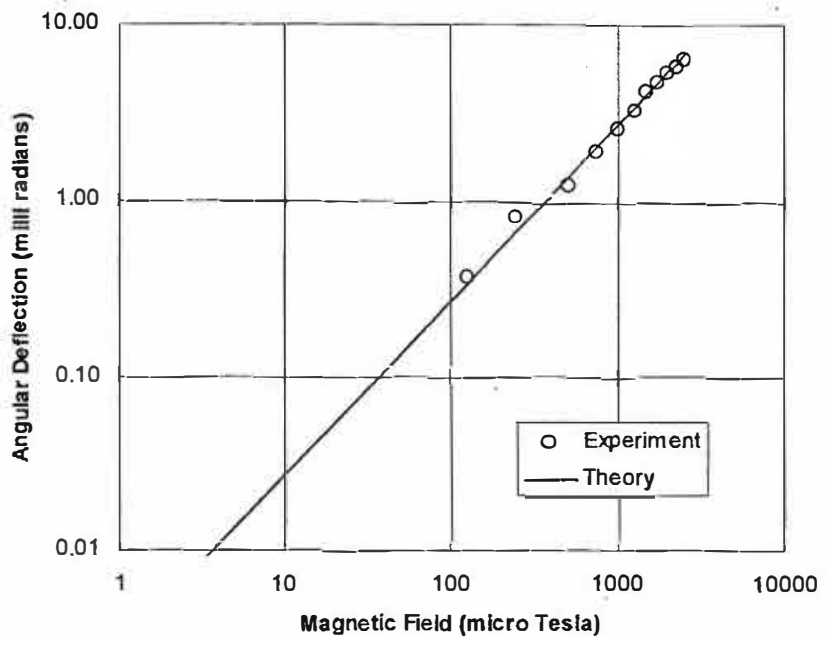

Figure 3. Comparison of experimentally determined angular deflections and theoretical calculations.

\section{CONCLUSIONS}

A novel micromechanical magnetometer can be realized by integrating ferromagnetic materials with polysilicon flexures. We have demonstrated the feasibility of this technology by designing, fabricating, and testing prototype devices that achieve a scaleindependent performance of $100 \mu \mathrm{T} / \mathrm{mrad}$. We have developed a simple model that adequately predicts out experimental results. By increasing the remnant magnetization, the volume of the magnetic material, and its aspect ratio, a 100-fold improvement in device performance is obtainable. In addition, by using capacitive displacement detection ICs, such as those used in inertial microsensors, a fully integrated MEMS magnetometer could be designed to detect $\sim 100 \mathrm{pT}$ with less than $1 \mathrm{~mW}$ of power.

\section{REFERENCES}

1. K. Bult, et. al, "Wireless Integrated Microsensors," Technical Digest Solid-State Sensor and Actuator Workshop, (Hilton Head '96), Hilton Head Island, SC, June 1996, pp. 205-210.

2. J. Lenz, "Review of Magnetic Sensors," Proceedings of the IEEE, vol. 78, no. 6, pp. 973-989, June 1990.

3. B. Eyre, K.S.J. Pister, and W. Kaiser, "Resonant mechanical magnetic sensor in standard CMOS", IEEE Electron Device Letters, vol. 19, (no. 12), pp. 496-498, 1998.

4. D.K. Wickenden, R.B. Givens, R. Osiander, J.L. Champion, D.A. Oursler, T.J. Kistenmacher, "MEMS-based resonating xylophone bar magnetometers", Proceedings of the SPIE, vol. 3514, pp. 350-358, 1998.

5. Cronos Integrated Microsystems: http://www.memsru.com/. 6. Jack W. Judy, Richard. S. Muller, and Hans. H. Zappe, "Magnetic microactuation of polysilicon flexure structures," IEEE Journal of Microelectromechanical Systems, vol. 4, no. 4, pp. $162-$ 169, 1995.

7. Jack W. Judy and Richard. S. Muller, "Magnetic microactuation of torsional polysilicon structures," Sensors and Actuators (Physical A), vol. 53, no. 1-3, pp. 392-397, 1996.

8. Jack. W. Judy and Richard. S. Muller, "Magnetically Actuated, Addressable Microstructures" IEEE Journal of Microelectromechanical Systems, vol. 6, no. 3, pp. 249-256, 1997. 